\title{
Characterization of etch pits found on a large-grain bulk niobium superconducting radio-frequency resonant cavity
}

\author{
Xin Zhao, ${ }^{1}$ G. Ciovati, ${ }^{1, *}$ and T. R. Bieler ${ }^{2}$ \\ ${ }^{1}$ Thomas Jefferson National Accelerator Facility (Jefferson Lab), 12000 Jefferson Avenue, Newport News, Virginia 23606, USA \\ ${ }^{2}$ Chemical Engineering and Materials Science, Michigan State University, East Lansing, Michigan 48824, USA \\ (Received 30 November 2009; revised manuscript received 15 November 2010; published 15 December 2010)
}

\begin{abstract}
The performance of superconducting radio-frequency (SRF) resonant cavities made of bulk niobium is limited by nonlinear localized effects. Surface analysis of regions of higher power dissipation is thus of intense interest. Such areas (referred to as "hotspots") were identified in a large-grain single-cell cavity that had been buffered-chemical polished and dissected for examination by high resolution electron microscopy, electron backscattered diffraction microscopy (EBSD), and optical microscopy. Pits with clearly discernible crystal facets were observed in both "hotspot" and "coldspot" specimens. The pits were found in-grain, at bicrystal boundaries, and on tricrystal junctions. They are interpreted as etch pits induced by crystal defects (e.g. dislocations). All coldspots examined had a qualitatively lower density of etch pits or relatively smooth tricrystal boundary junctions. EBSD mapping revealed the crystal orientation surrounding the pits. Locations with high pit density are correlated with higher mean values of the local average misorientation angle distributions, indicating a higher geometrically necessary dislocation content. In addition, a survey of the samples by energy dispersive x-ray analysis did not show any significant contamination of the samples' surface. The local magnetic field enhancement produced by the sharp-edge features observed on the samples is not sufficient to explain the observed degradation of the cavity quality factor, which starts at peak surface magnetic field as low as $20 \mathrm{mT}$.
\end{abstract}

DOI: 10.1103/PhysRevSTAB.13.124701

PACS numbers: 81.65.Ps

\section{INTRODUCTION}

Superconducting radio-frequency (SRF) resonant cavities made of high-purity bulk niobium are the basis for particle accelerator facilities that are commissioned for nuclear physics and high-energy physics study, e.g., the Large Hadron Collider (LHC/CERN), Continuous Electron Beam Accelerator Facility (CEBAF/Jefferson Lab), and the Spallation Neutron Source (SNS/Oak Ridge National Laboratory). SRF technology based on bulk niobium will be adopted in future accelerator facilities, such as advanced light sources and the International Linear Collider (ILC). The typical surface preparation of an SRF cavity made of high-purity (residual resistivity ratio of about 300) bulk $\mathrm{Nb}$ involves the removal of about $120-150 \mu \mathrm{m}$ of material from the inner cavity surface after fabrication by either buffered-chemical polishing (BCP) or electropolishing (EP). It has been demonstrated that EP allows achieving higher accelerating gradients $\left(E_{\text {acc }}\right)$ than $\mathrm{BCP}$ and is the treatment of choice for cavities in high-energy accelerators such as ILC [1]. SRF accelerator cavities prepared by either EP or BCP frequently show a quality-factor $\left(Q_{0}\right)$ drop (commonly referred to as " $Q$ drop" [2]) at accelerating field gradients above $\sim 20 \mathrm{MV} / \mathrm{m}$ (peak surface magnetic fields, $B_{p}$, above $\sim 100 \mathrm{mT}$ ). The causes of this phenomenon have not yet

\footnotetext{
*Corresponding author. gciovati@jlab.org
}

been fully elucidated. In addition, anomalous radiofrequency (rf) energy losses have been observed occasionally in the medium-field range $\left(E_{\mathrm{acc}}=5-20 \mathrm{MV} / \mathrm{m}\right)$, causing a strong so-called "medium-field $Q$ slope." A pronounced decrease of $Q_{0}$ in the medium-field range is also typical for cavities made of thin Nb films [3] and less common in cavities made of bulk Nb. Understanding the circumstances and mechanisms which cause enhanced rf losses in this medium-field range is important for the development of continuous wave $(\mathrm{CW})$ superconducting accelerators. To seek further insights that may account for these losses, a surface analysis study was undertaken on samples cut from a single-cell cavity made of large-grain $\mathrm{Nb}$ which exhibited a strong medium-field $Q$ slope. This provides an opportunity to investigate possible sources of enhanced rf losses. Samples were cut from locations where anomalous heating was detected by thermometry (hotspots) as well as from locations with negligible overheating (coldspots).

\section{EXPERIMENTAL METHODS}

The investigated $1.5 \mathrm{GHz}$ SRF single-cell cavity was made of a large-grain plate sliced from an ingot (ingot B) produced by the Companhia Brasileira de Metalurgia e Mineração (CBMM, Sao Paulo, Brasil). Ingot B had several large grains in the plane of the slice. The residualresistivity ratio (RRR) value of the material was $\sim 280$ with a tantalum content of $\sim 800 \mathrm{wppm}$. Results on other single 
cells made of this material are reported in [4]. The cavity had the following process history: (i) large-grain sheets sliced from ingot " $\mathrm{B}$ " (CBMM) by wire electric discharge machining (EDM); (ii) a deep-drawing process deforming the plates to half-cells; (iii) prior to the equator weld, $\sim 10$ min etching by $\mathrm{BCP}$ with $\mathrm{HNO}_{3}, \mathrm{HF}, \mathrm{H}_{3} \mathrm{PO}_{4}$ 1:1:1 by volume; (iv) electron-beam welding was done in two passes, inside and outside; joining the two half cells at the midplane; (v) after welding, the weld region was mechanically ground on the inside; (vi) the beam tubes were electron-beam welded on each side from the outside; (vii) the cavity was heat treated at $600^{\circ} \mathrm{C} / 10 \mathrm{~h}$, then etched to remove $\sim 10 \mu \mathrm{m}$ plus an additional $60 \mu \mathrm{m}$ by BCP 1:1:1 before the first rf test.

Buffered-chemical polishing was used to remove a damaged layer from the inner cavity surface after EDM cutting, die deforming, and mechanical grinding. During polishing, phosphoric acid acts as a buffer, and nitric acid as an oxidizing agent which produces niobium pentoxide $\left(\mathrm{Nb}_{2} \mathrm{O}_{5}\right)$. Hydrofluoric acid reacts with $\mathrm{Nb}_{2} \mathrm{O}_{5}$ and produces soluble $\mathrm{Nb}$ fluoride or $\mathrm{Nb}$ oxifluoride. The results of the first rf test at $1.7 \mathrm{~K}$ are shown in Fig. 1.

The quality factor, $Q_{0}$, monotonically decreased for increasing peak surface magnetic field, $B_{p}$, up to $68 \mathrm{mT}$, where thermal breakdown ("quench") occurred. The cavity performance did not improve significantly after an additional $30 \mu \mathrm{m}$ etching by BCP 1:1:1 (2nd rf test). In order to identify areas of the cavity with enhanced rf losses, an array of 576 resistor-temperature devices (RTDs) were attached to the outer cavity surface to measure the local temperature while increasing the rf field inside the cavity. Details on the thermometry system can be found in Ref. [5]. Temperature mapping showed the quench location to be on the cell's sidewall, closer to the iris. A large defect ("hole") was visible by the naked eye in that area. Repair of the defect was attempted by mechanical

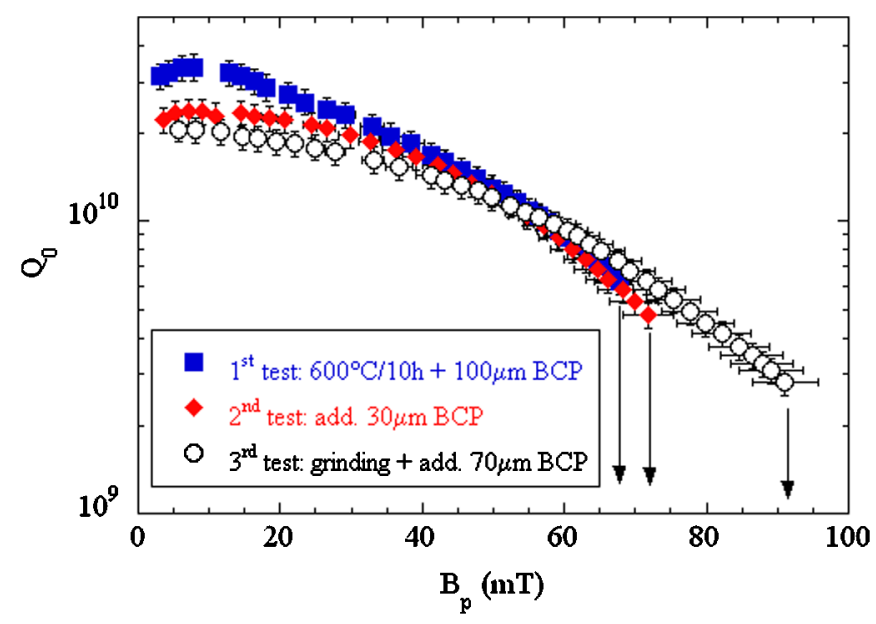

FIG. 1. $Q_{0}$ vs $B_{p}$ at $1.7 \mathrm{~K}$ after successive material removal showing a strong medium-field $Q$ slope. grinding, followed by an additional $\sim 70 \mu \mathrm{m}$ removal by BCP 1:1:1. The following rf test showed an improvement of the quench field, from 72 to $92 \mathrm{mT}$, and the quench occurred at the same location as before. The $Q_{0}$ vs $B_{p}$ curve was still characterized by a strong medium-field $Q$ slope. No field emission was detected during any of the rf tests. Figure 2 shows an "unfolded" temperature map just before the quench during test No. 3. Several "hotspots" are visible in the equator area. The location of the hotspots did not change significantly with successive material removal by BCP.

Twelve $12 \mathrm{~mm} \times 12 \mathrm{~mm}$ samples were cut from locations on the cavity shown in Fig. 2 by milling, using water as coolant. A $3.175 \mathrm{~mm}$ diameter powdered metal end mill was used. The samples were cut at a speed of about $7.62 \mathrm{~cm} / \mathrm{min}$ and the rotation speed of the end mill was about $600 \mathrm{rpm}$. For comparison, three coupons were from normal areas (coldspots No. 6-8), eight from hotspot zones, and one included the quench location near the iris (No. 2). Optical (HiRox ${ }^{\mathrm{TM}}$ KH-1300) and Hitachi 4700 field emission scanning electron microscopes (SEM) were used to observe the surface morphology. The densities of pits were counted for six samples (Nos. 1, 2, 5, 6, 7, and 8), three "coldspots" and three hotspots using optical microscopy at magnification 350 and 75. For each sample, pits were counted at 12 evenly distributed sites. At high magnification, the size of each survey site was $875 \times 690 \mu \mathrm{m}^{2}$, while it was about $4 \times 3 \mathrm{~mm}^{2}$ at low magnification, therefore covering the entire sample. Measurements at low magnification were done to measure the density of pits on separate grains in samples other than single crystals. Because at low magnification it is difficult to see pits smaller than about $50 \mu \mathrm{m}$ and to distinguish them from small scratches, we estimate the accuracy of the

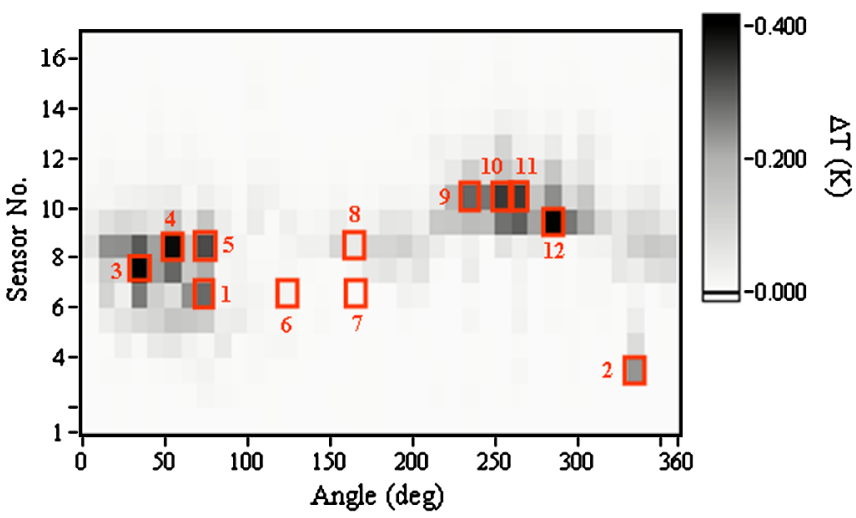

FIG. 2. "Unfolded" temperature map at $1.7 \mathrm{~K}$ at the highest field achieved in rf test No. 3, just before quench. Sensors No. 1 and No. 16 are located at the top and bottom iris of the vertically mounted cavity, respectively, while the equator weld is between sensors No. 8 and No. 9. Twelve samples, nine from hotspots and three from coldspots, with locations indicated by a red box, were cut from the cavity by milling for surface analysis. 
density of pits on each grain to about $15 \%$. The surface profile of several $1 \mathrm{~mm} \times 1 \mathrm{~mm}$ areas of each sample was measured with a $3 \mathrm{D}$ profilometer (KLA-Tencor ${ }^{\mathrm{TM}}$ ) using a $0.2 \mu \mathrm{m}$ diameter tip and a $1 \mathrm{mg}$ force.

The electron backscattered diffraction (EBSD) technique was used to investigate the crystal orientation and defect structure. The probing depth of EBSD is $\sim 30 \mathrm{~nm}$, which is in the same range as the rf penetration depth $(\sim 40 \mathrm{~nm})$. Therefore, the state of the crystal measured by EBSD is directly relevant to the material's SRF performance. Crystal orientations of the samples are shown using sample normal direction inverse pole figure maps generated with the TSL/EDAX orientation imaging microscopy $\left(\mathrm{OIM}^{\mathrm{TM}}\right)$ analysis software. The EBSD measurements were made using TSL/EDAX ${ }^{\mathrm{TM}}$ systems installed on electron microscopes at Virginia Tech, University of Maryland, College Park, and NASA Langley. Some of the SEM images are expanded versions of tilted images obtained to identify OIM scans. OIM maps are shown in two modes, where low confidence pixels are overlaid with shades of gray that black out low confidence pixels, or in some cases, they were cleaned up using a neighbor correlation criterion that changed a pixel orientation with a low indexing confidence to a neighboring orientation with a high confidence index.

\section{RESULTS}

Other than the mechanical scratches attributed to the dissection process, the most obvious surface characteristics are "etch pits" (Figs. 3-10) along bicrystal boundaries [Figs. 3(b), 5(c), and 7(b)], tricrystal junctions (Figs. 6-8), and in the middle of a grain interior [Figs. 4(c) and 9(b)]. The etch pits have a common geometrical feature: they have clear crystal facets. The size of the pits, measured by profilometry, ranged between $20-80 \mu \mathrm{m}$ in width and $2-10 \mu \mathrm{m}$ in depth. The etch pits are not uniformly distributed. The average pit density, measured at high magnification, and features of these samples are listed in Table I. All the coldspot samples (Nos. 6, 7, and 8) have a lower pit density than the hotspots (Nos. 1, 2, and 5).

Figures 3 and 4 show optical microscopy and SEM images which illustrate the nonuniform pit distribution among grains of different orientation and the pit geometries. The images shown in Fig. 3 were taken on sample No. 9. They show a higher pit density along a grain boundary than in the peripheral domain; the grain on the right side of Fig. 3(b) obviously has higher pit density than the left grain. The images shown in Fig. 4 were taken on sample No. 5. In this figure, there are more elongated pits on the grain labeled as "III" than in the neighboring grains, "I" and "II". The reason for the "elongated" shape is unknown.

Samples 3, 5, 7, and 9 each consist of three large crystals. Etch pits were found at all the tricrystal junctions (Figs. 6-8). At the junction, the profile of the etch pit of
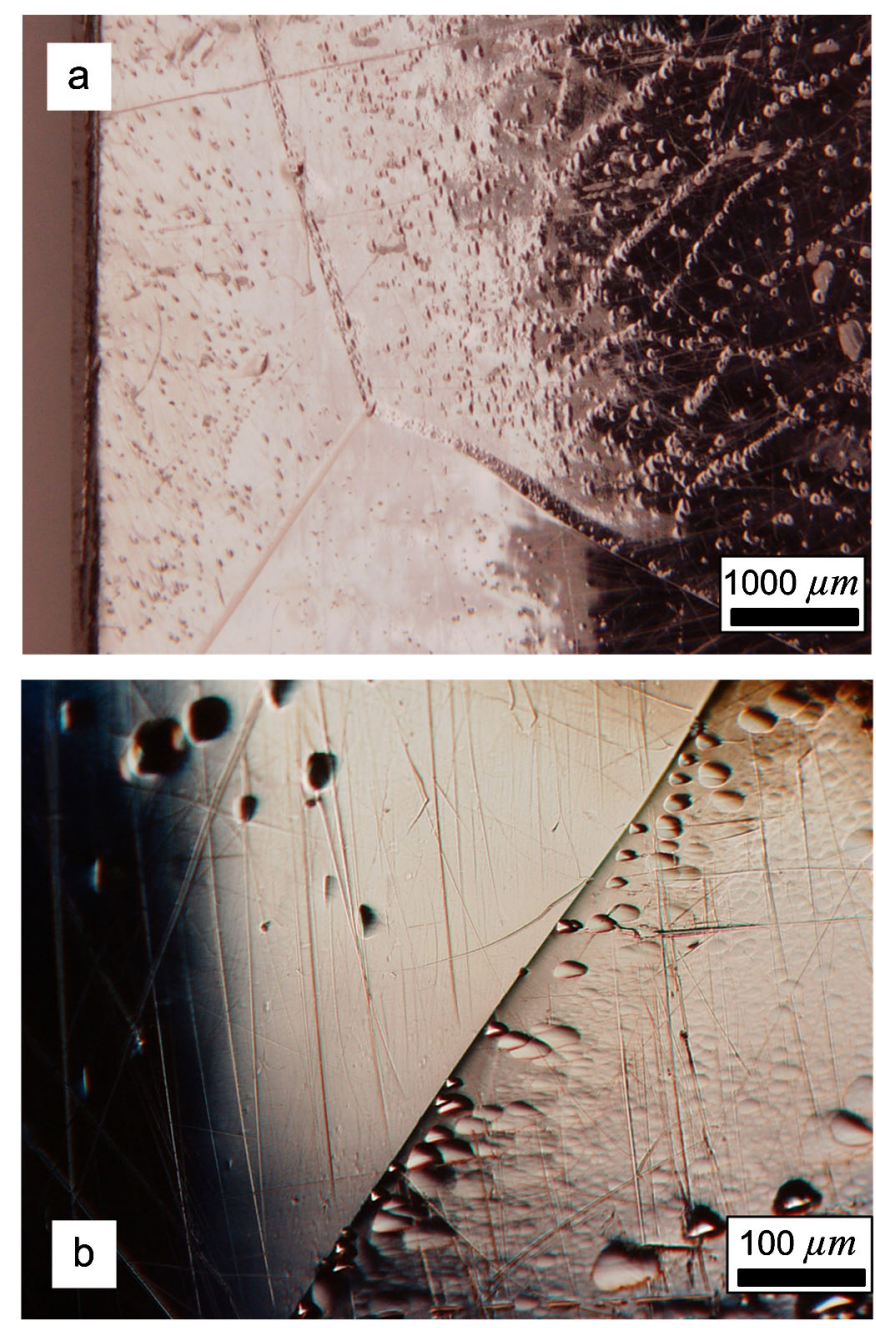

FIG. 3. Optical microscopy images of sample No. 9, showing high density of etch pits (a) and nonuniform pit distribution (b) on different crystal grains.

sample No. 7 (a coldspot sample) is much shallower than the others (Fig. 6). Other etch pits at the tricrystal junctions are deep, sharp, and have many facets (Figs. 7 and 8). Figure 7 shows the etch pit at the tricrystal junction of sample No. 9. The pit has sharp and deep features. The EBSD technique was utilized to map the crystal orientation surrounding the tricrystal junction. The orientations are visualized in the orientation map in Fig. 7(c) with superposed prisms showing the actual crystal orientation, and slip system planes and directions with high resolved shear stress are indicated with the Schmid factor $m$, as discussed later. Figure 8 shows the tricrystal etch pit on sample 5. The pit diameter is $\sim 5 \mu \mathrm{m}$ and the radius of curvature of its top edge is quite sharp, $\sim 50 \mathrm{~nm}$, as observed in the SEM image. Thus its aspect ratio, defined as the ratio of the pit diameter divided by the curvature radius at the edge of the pit, is $\sim 100$.

Energy dispersive x-ray analysis performed on all samples did not show any significant contamination of 

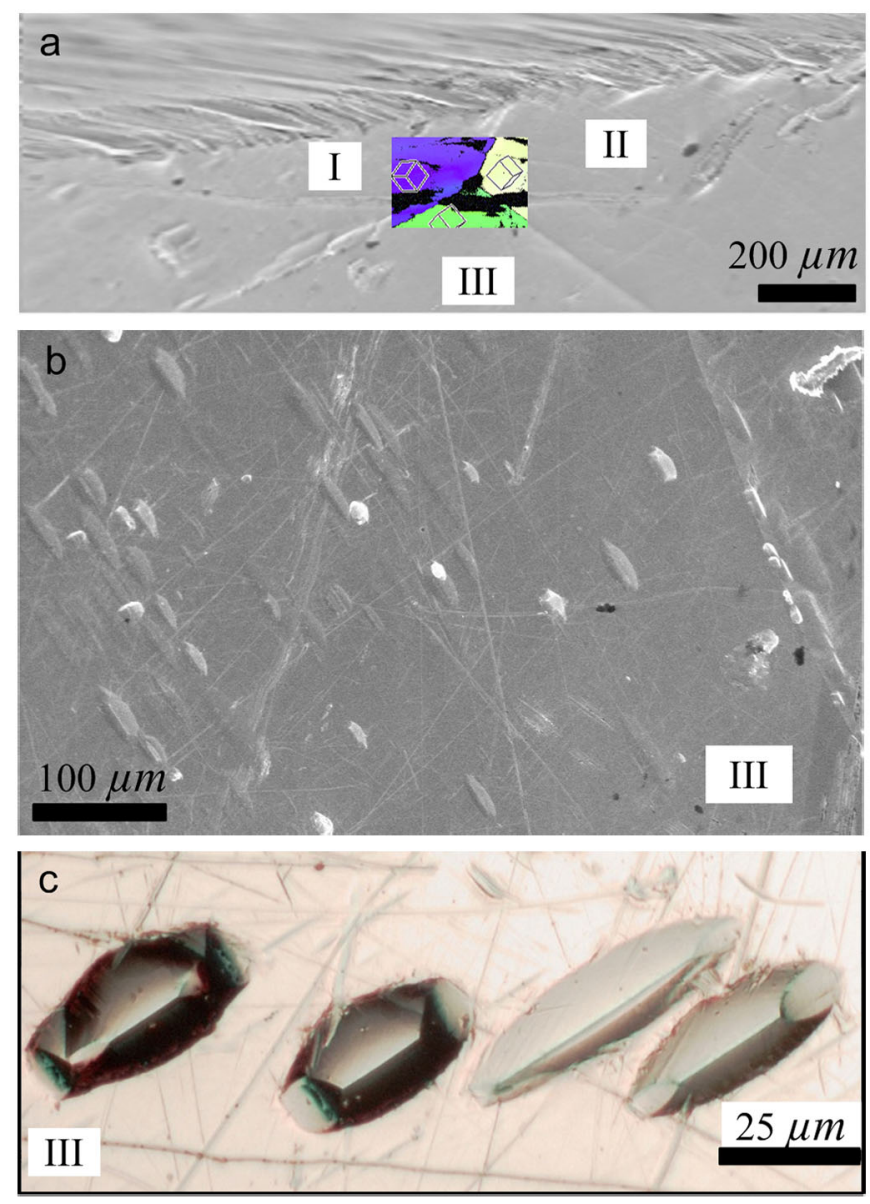

FIG. 4. Pits distributed nonuniformly on the three grains found on sample No. 5. The SEM image with overlapping EBSD orientation map is shown in (a). SEM images with greater magnification (b), (c) shows the elongated feature of the pits found on grain labeled "III".

the $\mathrm{Nb}$ surface. The areas surrounding and inside a pit have been analyzed by Auger electron spectroscopy, also indicating no impurities.

Figure 9 shows an example of etch pit lines on a single grain, as observed on sample 3. Crystal orientation obtained from the orientation map in the surrounding area shows some alignment of dislocations with slip traces on $\{110\}$ planes, which suggests more of an edge character [an edge dislocation line direction is perpendicular to the Burgers vector indicated by a blue arrow in Fig. 9(b)]. Figure 10 shows an optical micrograph of sample 1 at low magnification, showing etch pits in a networked pattern which could be indicative of subgrain boundaries caused by deformation and heat treatment.

Figures 11 and 12 present the twelve samples' crystal orientations in inverse polar figure maps and inverse pole figures. No correlation of hot/cold spot character with crystal orientation is immediately apparent. It is clear that some peaks in the inverse pole figures are sharp and others are spread out, indicating considerable orientation
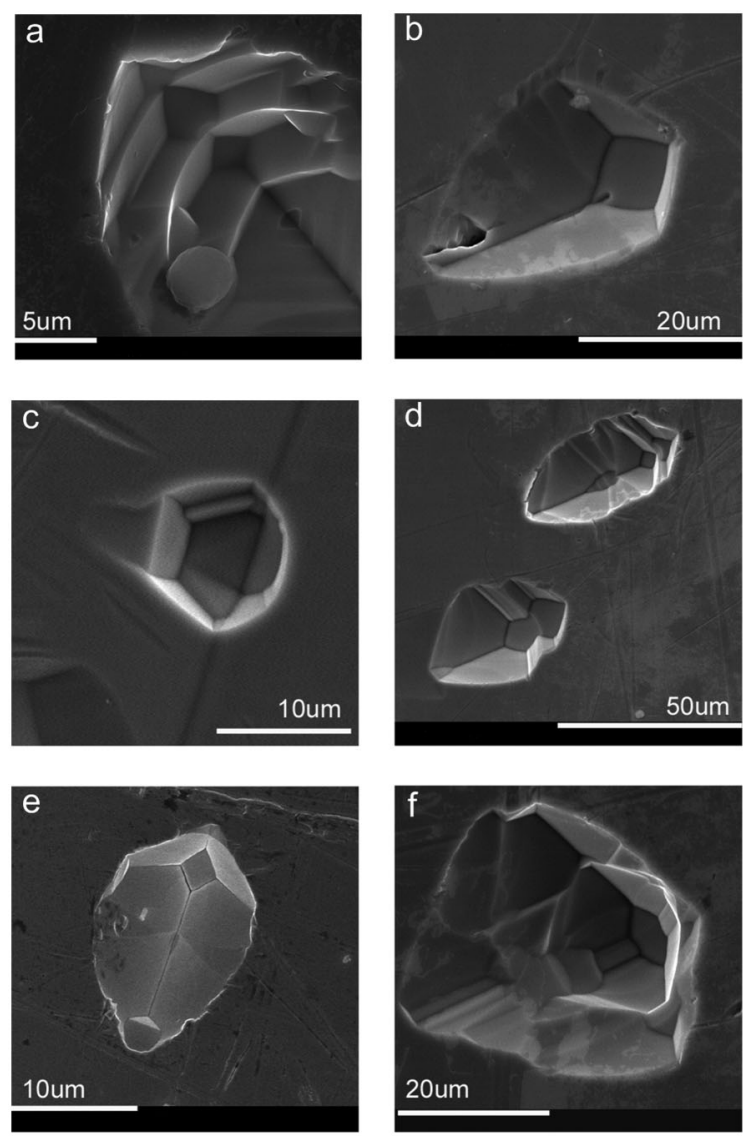

FIG. 5. SEM images of pits which have complex symmetrical geometry observed on samples No. 2 (a), No. 8 (e), and No. 1 (b), (c), (d), (f).

gradients in the grain. Our future work will further explore the relationship between crystal grain orientation and the pit formation mechanism.

Following the observations and methodology of Romanenko [6], the EBSD data was analyzed to obtain, for each grain in each sample, a distribution of the local average misorientation angle, $\theta$. In this analysis we considered the local average misorientation between a pixel and up to its 8th neighbor. These $\theta$ distributions are well fitted with the Gaussian distribution function (fit correlation factor, $r^{2}>0.8$ ) and the values of the mean for each grain are plotted in Fig. 13 for all the samples. The error bars correspond to the standard deviation of the mean for each distribution. The average etch pit density for each grain, $N_{\text {avg grain }}$, is also plotted, showing a correlation between etch pit density and $\theta_{\text {mean }}$ among different grains on the same sample, but not among the various samples.

In at least three samples with more than one crystal orientation (1, 4, and 9), the EBSD data were taken in regions of the samples where the corresponding SEM images show grains with a different pit density. In all three cases, the grain with the lowest value of $\theta_{\text {mean }}$ also has fewer pits, compared to the neighboring grains on the 

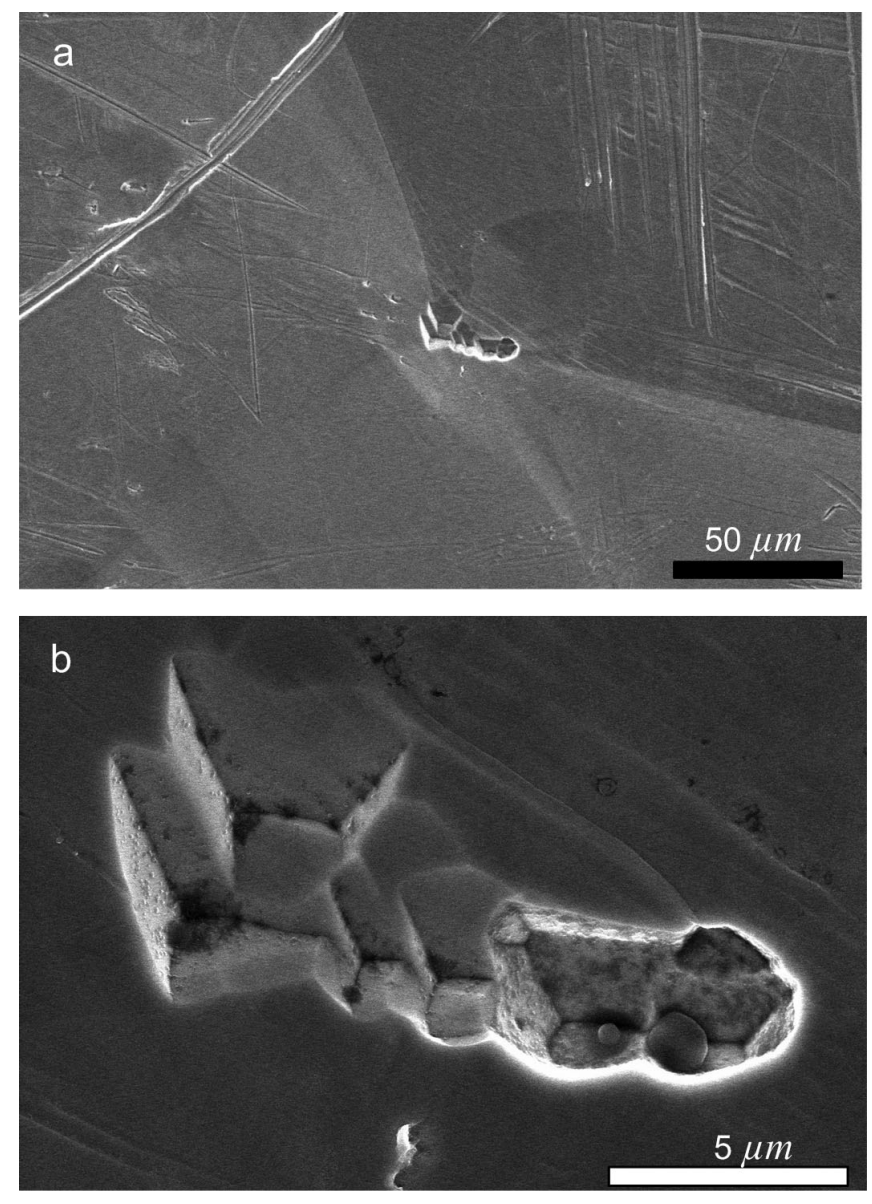

FIG. 6. SEM image showing the tricrystal etch pit of sample No. 7 (a). A higher resolution image of the pit is shown in (b). The profile of the pit is shallower than the ones shown in Figs. 7 and 8 .

sample and consistently with the data shown in Fig. 13. This is shown, for example, in Fig. 14 for sample No. 1.

Samples 3 and 9 were examined in greater detail to identify potential relationships between etch pits and activated slip systems. As the local stress state is not known with certainty, and the orientation of the samples with respect to the forming history was not intentionally identified, a working assumption of biaxial tension was assumed (i.e. a stress tensor with $\sigma_{x x}=\sigma_{y y}=$ constant, with all other terms $=0$ ), which is independent of the orientation of the sample with respect to the cavity geometry. Based upon this, the Schmid factor $m$ for all 24 slip systems on $\{110\}$ and $\{112\}$ planes were computed, $m^{\alpha}=$ $\boldsymbol{\sigma}^{\prime}: \mathbf{P}^{\alpha} /\left\|\boldsymbol{\sigma}^{\prime}\right\|$, where $\boldsymbol{\sigma}^{\prime}$ is the global stress tensor expressed in the crystal coordinate system, and $\mathbf{P}^{\alpha}$ is the Schmid tensor for slip system $\alpha$. To find potential plane traces for each slip system, the slip plane normal was expressed in the laboratory coordinate system and the cross product with the sample normal direction $\left[\begin{array}{lll}0 & 0 & 1\end{array}\right]$ gives a vector that describes the slip trace; this direction was
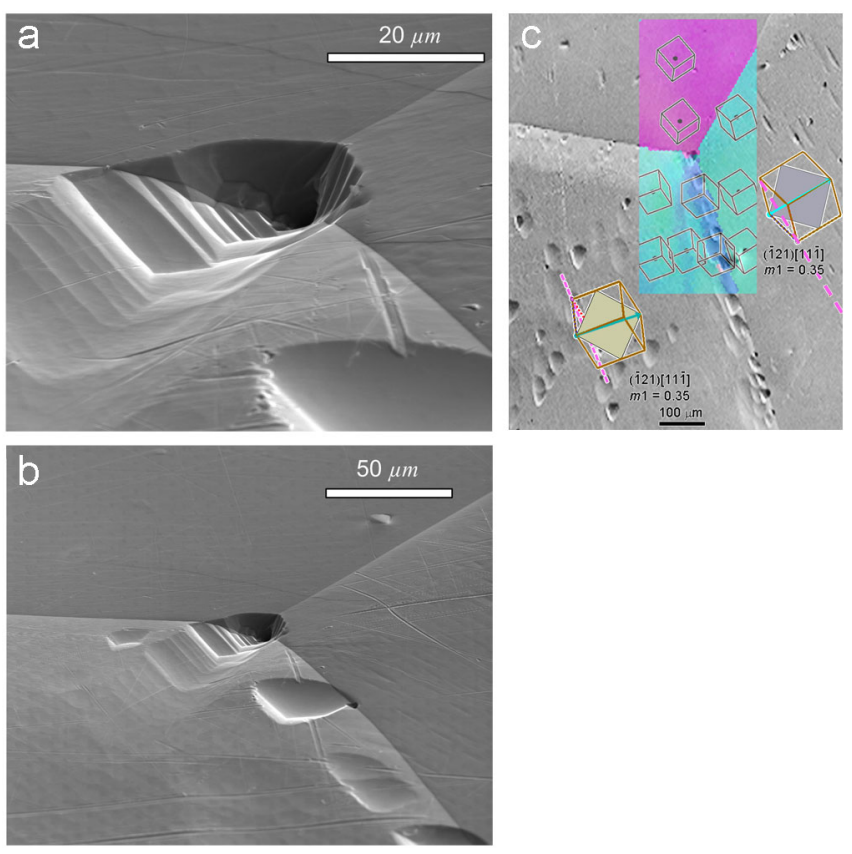

FIG. 7. Tilted SEM images showing an etch pit at a tricrystal junction of sample No. 9 (a), (b). The neighborhood of the magnified image in (a) is shown in (b). A normal direction crystal orientation map (untilted view) of the tricrystal junction (c) shows that etch pits in the lower two grains are consistent with screw dislocations where the line direction is parallel to Burgers vectors indicated with blue arrows on slip planes. The lighter plane has a normal direction that comes out of the surface and darker plane into the surface. The Schmid factor $m$ is indicated, e.g. $m 1=0.35$ is the highest resolved shear stress operating on a slip system in the grain, with a biaxial tension stress tensor.

compared with lines of etch pits. In most cases, a row of etch pits corresponded with a slip system that had one of the higher Schmid factors, such as the examples shown in Figs. 7, 9, 14, and 15. In Figs. 7(c) and 9(b), a unit cell is shown with a particular slip system identified, their rank (in order of highest to lowest Schmid factor), and the corresponding plane trace. Some micrographs showed very straight grooves that ended at grain boundaries that were clearly not scratches, and in many cases, these lined up with particular slip systems having high Schmid factors, as illustrated in Fig. 15.

The lower grain boundary in Fig. 7(c) has a band misoriented by $10^{\circ}$ from the left grain on the left side of the grain boundary (shown in darker blue) in which many etch pits are found. The two light blue grains on either side have nearly the same orientation with respect to the surface, and hence the same slip system is most highly stressed. They are related to each other by a rotation of $31^{\circ}$ about a $\langle 210\rangle$ axis. The darker band to the left of the boundary is misoriented by $40^{\circ}$ from the right grain about the same axis. In cubic crystals, coincident site lattice (low energy) boundaries exist with a $35.43^{\circ}, 40.88^{\circ}$, and $27.91^{\circ}$ with rotations 

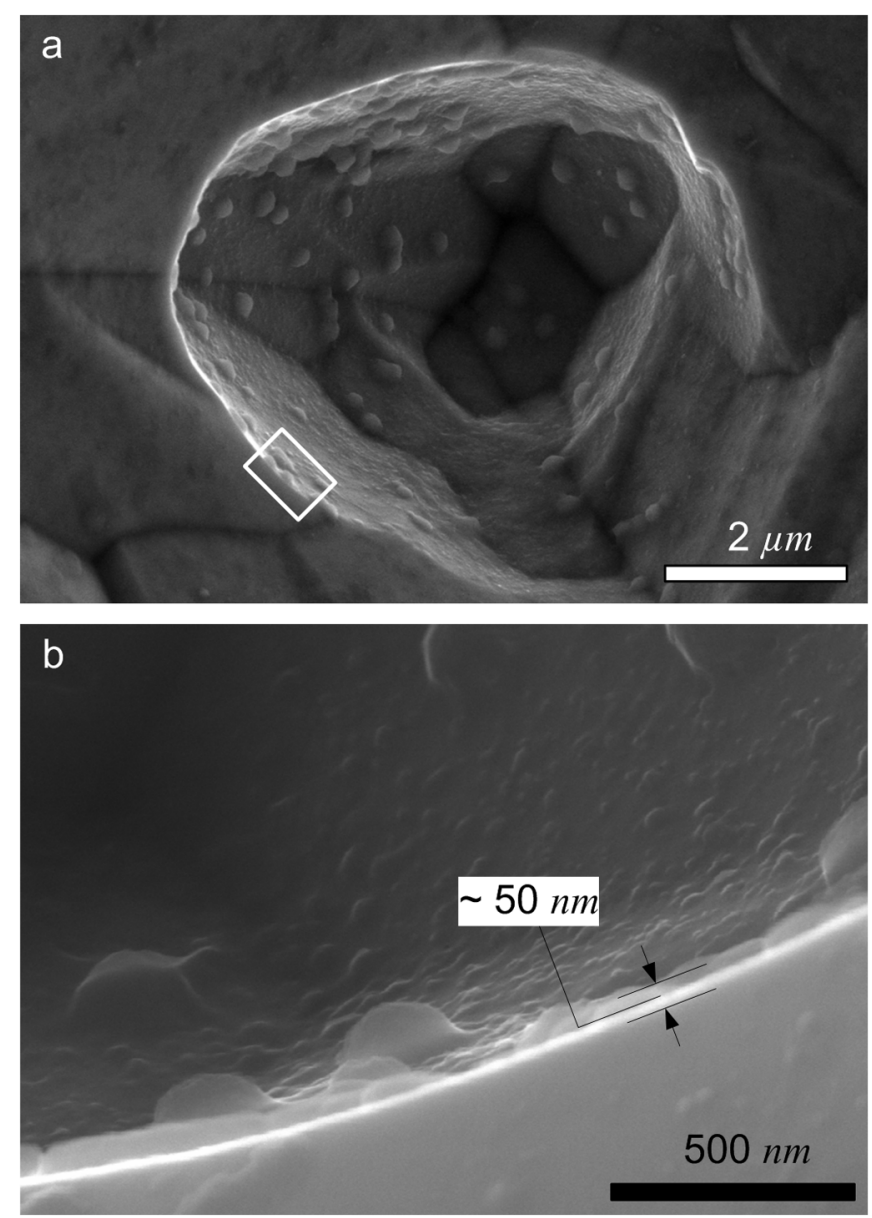

FIG. 8. SEM image showing the tricrystal etch pit found on sample 5. The pit has deep, sharp profiles. The pit diameter is $\sim 5 \mu \mathrm{m}$ and its edge curvature radius $\sim 50 \mathrm{~nm}$ as estimated from a higher resolution SEM images shown in (b).

about the $\langle 210\rangle$ axis $(\Sigma 27 b, \Sigma 41 b$, and $\Sigma 43 b$, respectively ${ }^{1}$ ). If the original boundary had the lowest energy of these three, $\Sigma 27 b$, then deformation and recovery may have caused a split of this originally low energy boundary into two different low energy boundaries, a $10^{\circ}$ boundary and a slightly higher energy $\Sigma 41 b$ boundary. If so, then the left grain must have deformed in a manner where the darker blue region to the left of the grain boundary rotated in the opposite direction as the rest of the grain on the left, implying that deformation constrained by this boundary differed from the nearby bulk crystal. Small deviations from the geometrically perfect $\Sigma$ relationship are

\footnotetext{
${ }^{1}$ The $\Sigma$ nomenclature describes the number of coincident lattice sites present in two adjacent lattices. For example, a $\sum 27 b$ misorientation has 1 in 27 sites in both lattices, which results in boundaries with periodic atoms belonging to both lattices and, hence, lower energy. The "b" refers to a $\langle 210\rangle$ axis of rotation. The boundary energy is lowered further when the boundary plane is a low index plane for both crystals, which makes the boundary "special" $[7,8]$.
}

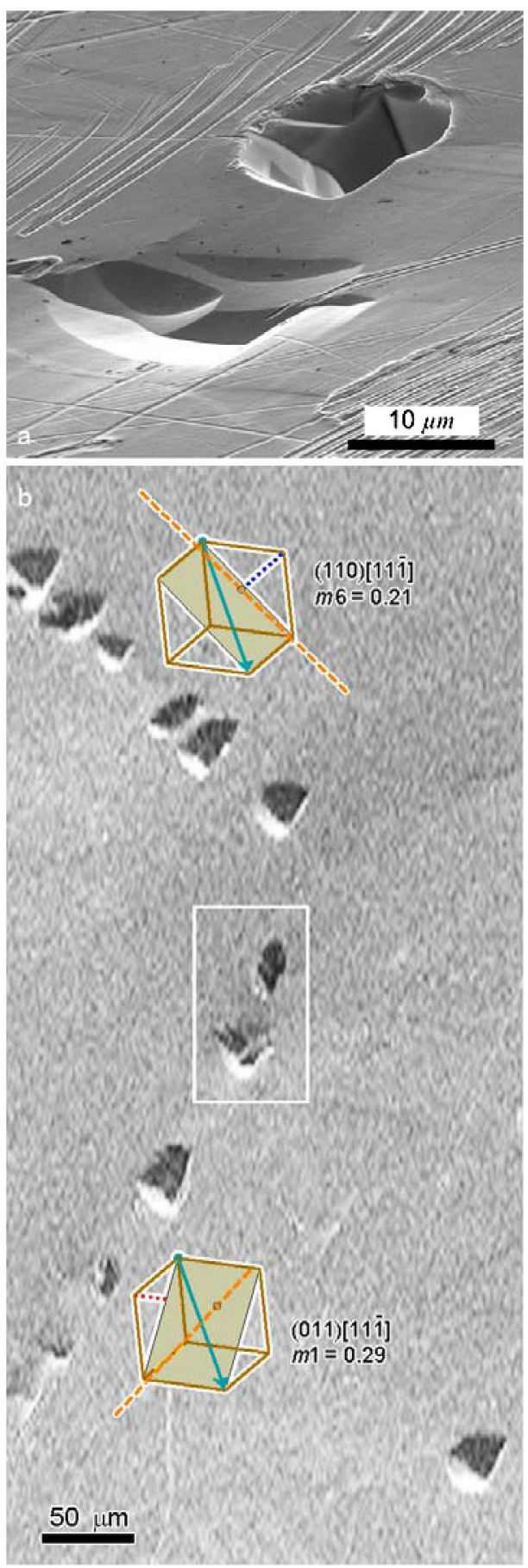

FIG. 9. (a) $70^{\circ}$ tilted SEM image of a single grain showing etch pits in sample No. 3. (b) SEM image of sample No. 3 at lower magnification. The prisms illustrate the highest $(m 1)$ and the 6th highest $(m 6)$ resolved shear stresses (as indicated in caption of Fig. 7). The area enclosed in the white box is shown in (a) at greater magnification and rotated by $70^{\circ}$.

accommodated with misfit dislocations that can accommodate up to a few degrees of misorientation and still maintain low boundary energy [9]. This effect is similar to a low angle boundary, and these misfit dislocations can be the 


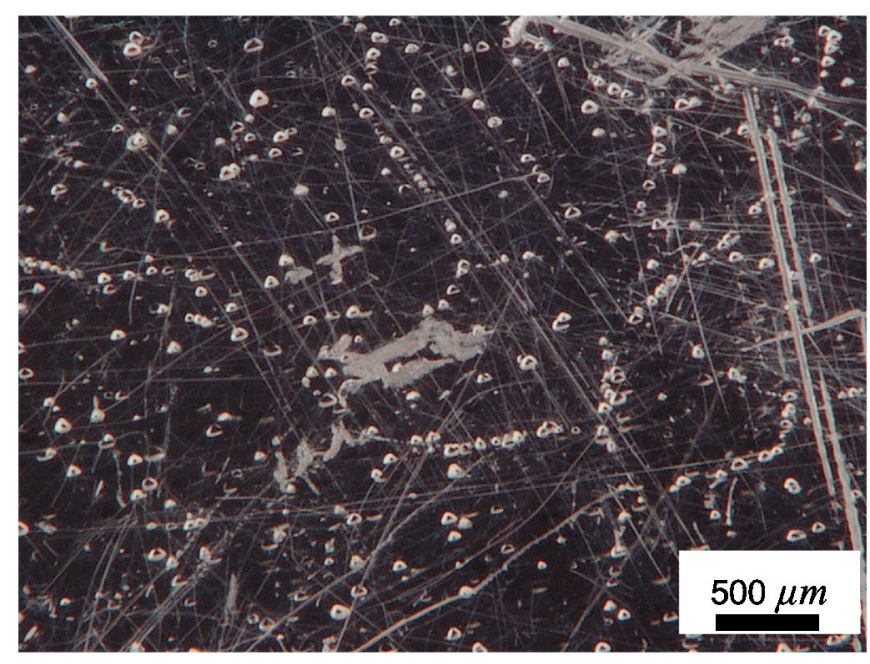

FIG. 10. Optical micrograph of sample 1. The pits' distribution has a "network" pattern that might reveal subgrain boundaries.

source of etch pits. The scenario described above is consistent with grain boundary engineering strategies and observations [7,8], which are extensively developed for face centered cubic polycrystals [7] and only some work has been done with this paradigm with body centered cubic polycrystals, e.g. [10].

\section{ANALYSIS AND DISCUSSION}

It is known that chemical etching processes on crystal defects (such as dislocation, stacking fault, and impurity segregation) can produce distinct types of preferential etching [11]. Etch pit geometry is determined by anisotropic etching rates.

Cai reported that the profile of an etch pit directly correlates with the orientation of an exposed crystal face [12]. It was explained that, since various crystal planes have different surface energies, they yield different etching rates. Anisotropic etching rates thus yield the symmetric and faceted features of the pits. Figure 3 in Ref. [12] illustrates how the geometrical features of tetrahedral etch pits are related to the crystal plane orientation exposed to an etchant.

Evans [13] described dislocation induced etch pits on large-grain $\mathrm{Nb}$ materials. His study showed that: (i) pits have distinct densities on various grains; (ii) pits tend to segregate on tricrystal grain junctions; (iii) pits tend to be distributed along a slip path; (iv) pits decorating a slip path can cross grain boundaries.

Other than the tetrahedral pits that were observed by Cai [12], this study shows that most pits have a complex geometry deviating from a tetrahedral profile. Figure 5 shows some pits sitting within a grain or on a bicrystal boundary that cannot be explained by Cai's model. His model (Fig. 3 in Ref. [12]) predicted that only tetrahedral etch pits could be produced by etching. The model was based on different etching rates of distinct crystal surface energy. The more complex geometry (nontetrahedral profile) observed in this study may result from inclined dislocations that do not intersect the surface in a manner that makes the surface normal to be parallel to a direction of symmetry.

For crystalline metallic materials, plastic deformation occurs by dislocation generation and multiplication. The dislocation distribution is determined by the activity of different slip systems and their intersections with each other. As different slip systems are activated in different grains according to the resolve shear stress on planes and directions, the spatial arrangement of dislocation will vary from grain to grain. Grain boundaries present barriers to propagation of dislocations, resulting in higher concentrations of dislocations (and hence, etch pits) near some grain boundaries. Thus, the dislocation pattern formed during the pressing of the half-cell may affect the etch pit features. Some etch pits are aligned in straight lines (Fig. 9) or in a networked fashion (Fig. 10). According to Evans (see Fig. 4 on page 258 in [13]) and Sangwal (see Fig. 8.8 on

TABLE I. Summary of surface features and density of etch pits found on samples dissected from a large-grain single-cell cavity.

\begin{tabular}{|c|c|c|c|c|}
\hline Sample No. & No. of grains & rf behavior & Pit density $\left(\right.$ No. $\left./ \mathrm{mm}^{2}\right)$ & Surface features \\
\hline 1 & 2 & Hotspot & 34.2 & \\
\hline 2 & 1 & Hotspot & 29.1 & Quench site \\
\hline 3 & 3 & Hotspot & $\ldots$ & \\
\hline 4 & 3 & Hotspot & $\ldots$ & \\
\hline 5 & 3 & Hotspot & 61.7 & $\begin{array}{l}\text { A very deep pit on tricrystal junction; high } \\
\text { density of elongated etch pits on }\{110\} \text { plane }\end{array}$ \\
\hline 6 & 1 & Coldspot & 6.3 & \\
\hline 7 & 3 & Coldspot & 19.7 & A shallow pit on tricrystal junction \\
\hline 8 & 1 & Coldspot & 17.6 & \\
\hline 9 & 3 & Hotspot & $\ldots$ & A very deep pit on tricrystal junction \\
\hline 10 & 1 & Hotspot & $\cdots$ & \\
\hline 11 & 1 & Hotspot & $\cdots$ & \\
\hline 12 & 2 & Hotspot & $\ldots$ & \\
\hline
\end{tabular}



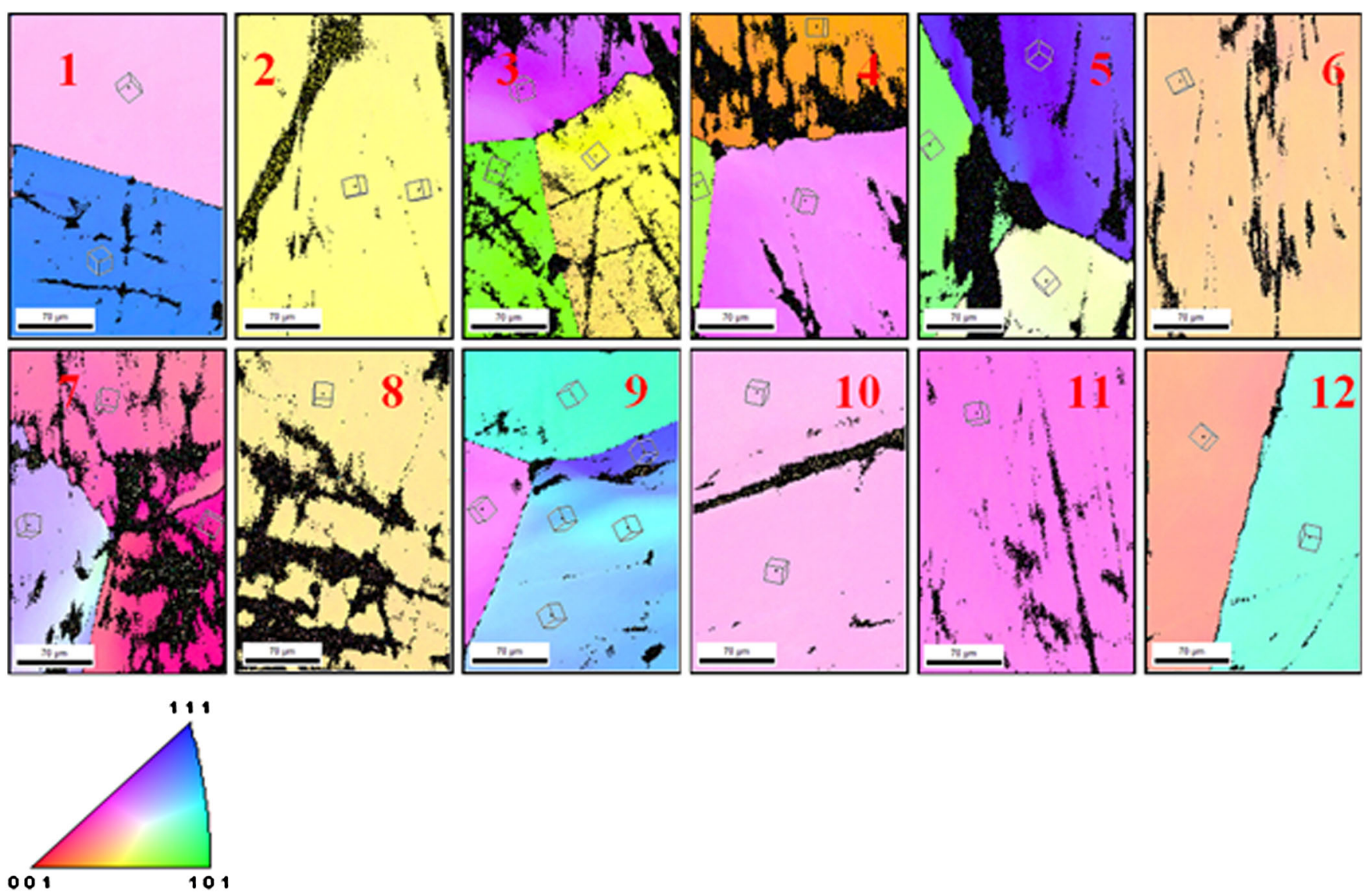

FIG. 11. Crystallographic orientation of the 12 samples measured by EBSD. Nb [001] inverse pole figure (IPF) color legend is shown on left bottom corner.

page 274 and Fig. 11.2 on page 374 in [11]), this pattern might be caused by multiple dislocations on particular slip systems.

After the deforming processes, the dislocation density is quite high, but the recovery heat treatment at $600^{\circ} \mathrm{C}$ enabled short range rearrangement of dislocations by glide and climb that reduced dislocation density. Recovery results in pairs of dislocations of opposite sign that are spatially close to each other to move toward each other and then disappear via joining of two half planes (statistically stored dislocations). The remaining unpaired (geometrically necessary) dislocations cannot annihilate so they can be isolated, as illustrated in Figs. 3(b), 9(b), and 10. If there are locally large densities of geometrically necessary dislocations, they can arrange themselves in energetically favorable structures called subgrain boundaries, by a process known as "polygonization." These results in lines of dislocations which could then be nucleation sites for etch pits, such as the line of pits shown in Figs. 3, 7, 9, and 10. As the dislocation line energy in $\mathrm{Nb}$ is relatively low
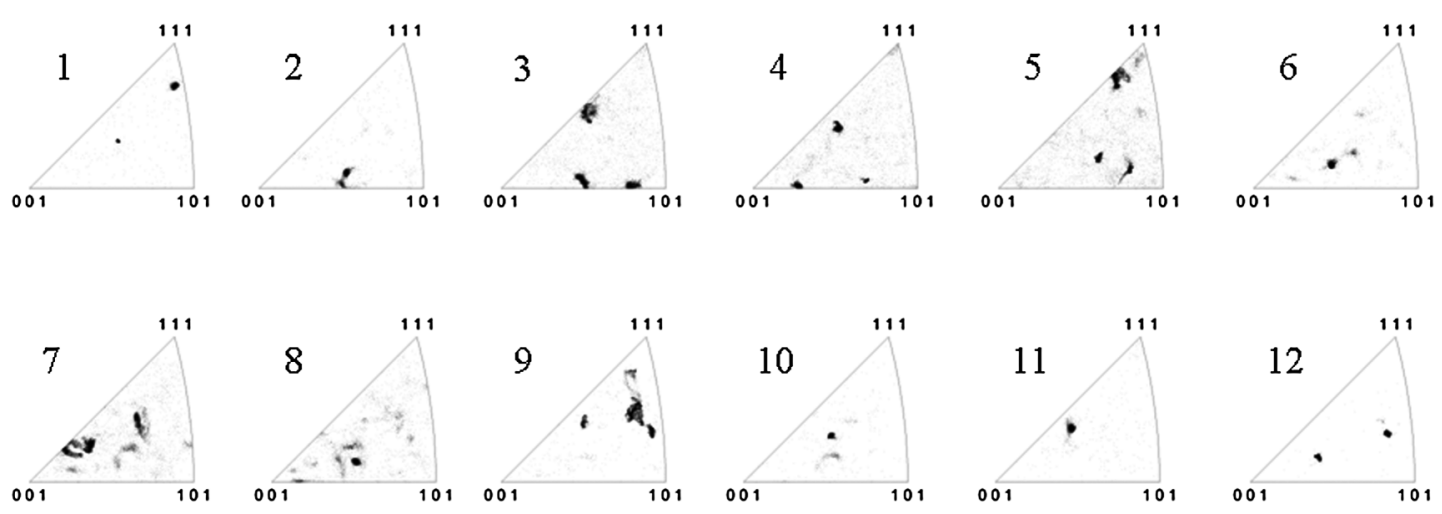

FIG. 12. Nb [001] IPF obtained by EBSD measurements on all samples. 


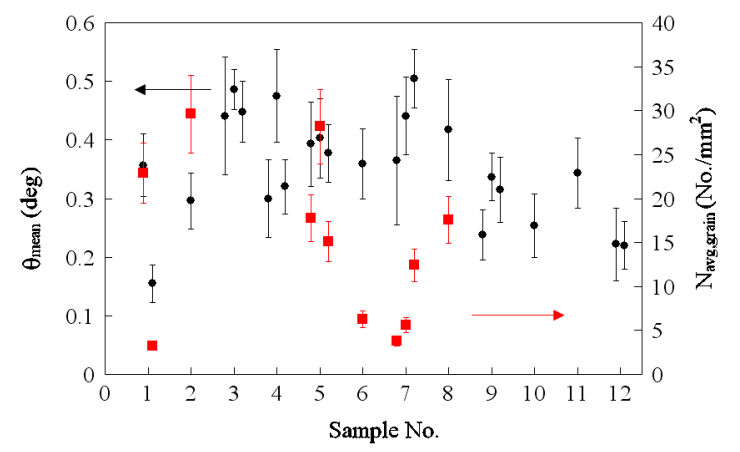

FIG. 13. Mean values of the local average misorientation distributions, obtained from the EBSD data on each grain for all the samples (black circles) and average density of pits measured on each grain for six samples (red squares). Samples with more than one grain have multiple points in the plot, corresponding to the different misorientation angle distributions for each grain. The $N_{\text {avg,grain }}$ values are plotted in the position correlated with the value of $\theta_{\text {mean }}$ for that grain.

[14], dislocations on slip bands can also be retained because they are geometrically necessary (if their opposite signed partners exist, they are widely separated on the other side of the grain). The retained dislocations on slip bands may account for instances of grooves or rows of pits that end at a grain boundary [Figs. 3(b) and 15] and are aligned with a highly stressed slip system (Figs. 7, 9, 14, and 15). In most of the cases where linear features were observed, they correspond with the more highly stressed slip systems. The actual state of stress present in a given crystal is difficult to know without a more thorough analysis, which will be explored and reported in future articles. Thus, with this preliminary work it appears to be possible, from the perspective of activated slip systems, to correlate grain orientations with propensity to develop pits, but the orientation of the dislocation line does affect the geometry of the pit (Figs. 4 and 5). The recovery heat treatment following modest amounts of deformation is a typical recipe for grain boundary engineering strategies [7] that favor development of low energy boundaries consisting of low angle and $\Sigma$ boundaries with misfit dislocations that are very stable, and difficult to remove.

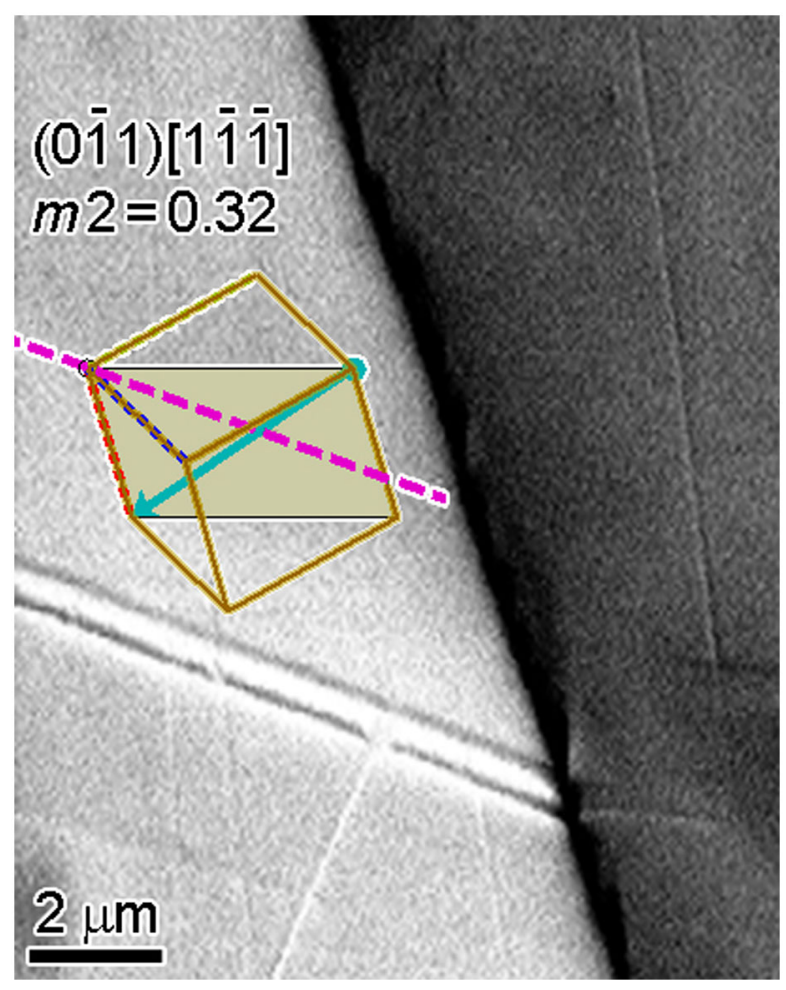

FIG. 15. Slip trace features on sample appear like very deep scratches, but they align well with the plane trace of the 2nd most highly stressed slip system in the grain as explained in the caption of Fig. 7 (they also do not continue in the same direction across grain boundaries), suggesting that a high density of dislocations were etched to make a groove.

As shown in Ref. [15], the local average misorientation angle is directly related to the geometrically necessary dislocation content (i.e. retained dislocations after recovery). As coldspots did not show distinctly different mean value of $\theta$ (samples 6, 7, and 8 in Fig. 13) than hotspots, there is no direct correlation between the rf performance and lattice curvature resulting from geometrically necessary dislocation content. This contrasts with the EBSD results on samples cut from bulk $\mathrm{Nb}$ cavities reported in [16] that showed a correlation between hotspot behavior at $B_{p}>90 \mathrm{mT}$ and higher $\theta$ values. Nevertheless, our analysis does show that higher density of pits is correlated

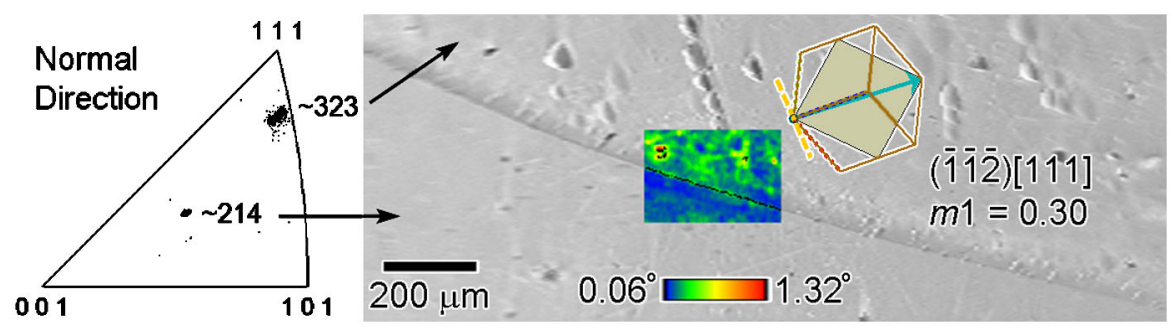

FIG. 14. The 3rd neighbor local average misorientation map for sample No. 1 overlapping the SEM image. The grain on the bottom with an orientation $\sim(214) \|$ surface has fewer pits and lower $\theta$ values (sharper spot on inverse pole figure) than the grain on the top with orientation $\sim(323) \|$ surface. The etch pits above the map correlate well with the plane trace of the most highly stressed slip system. 
with higher values of $\theta_{\text {mean }}$ (but there are also cases where a low pit density is measured for relatively high $\theta$ values, Fig. 13). This is consistent with dislocations acting as nucleation sites for etch pits, but dislocations are apparently necessary for pitting, but not a sufficient cause. As we could not establish a clear correlation between grain orientations and $\mathrm{rf}$ behavior, it is not possible to indicate, in absolute terms, a crystal orientation which is more susceptible to pitting. A possible reason for this, as shown in [14], is that slip system activation during forming of the halfcells depends on the crystal orientation, grain boundaries, and orientation gradients within a grain.

A more effective way to remove dislocations is by recrystallization, as dislocations are swept out by moving grain boundaries. Thus, there is a need to explore the effectiveness of a recrystallization anneal $\left(\geq 800^{\circ} \mathrm{C}\right.$ ) on removing dislocations in large-grain cavities. Also, it will be valuable to examine what slip systems are activated, and to determine how the geometrical arrangement of dislocations affects pitting. Initial studies on large-grain singlecell cavities heat treated at $800^{\circ} \mathrm{C} / 3 \mathrm{~h}$, without subsequent BCP, showed improved $Q_{0}$ values over baseline rf tests, after BCP [17].

It has been shown both by experiments [18] and simulation [19] that magnetic field enhancements at sharp edges, such as those found on pits, may lead to early local breakdown of the superconducting state, which may or may not be thermally stable. The rf and temperature measurements of this cavity indicate increasing nonlinear losses starting at very low field $\left(B_{p} \sim 20 \mathrm{mT}\right)$. The onset of such losses at these surface fields is unusual. More typically, strong nonlinear effects appear only above $\sim 90 \mathrm{mT}$ (so-called "high-field $Q$ slope" or " $Q$ drop" [1]). Applying the hypothesis of local quenches at sharp edges, as suggested by Knobloch et al. [20], starting at such low field would imply a field enhancement factor of about 10 in order to create a local $B_{p}$ approaching the critical field of $180 \mathrm{mT}$. According to the analysis of Shemelin [19], such values of the field enhancement factor would require the presence of pits with aspect ratio of about 1000. The use of a high resolution SEM enabled us to identify pits with sharp edges but with aspect ratio of only about 100 . Therefore it seems that losses occurring at such low field may not be simply explained by local magnetic field enhancement. The $\mathrm{Nb}$ samples have been analyzed by point contact tunneling (PCT) spectroscopy at Argonne and preliminary data show unusual features in the conductance curves of hotspot samples, particularly near grain boundaries [21]. The possibility of other loss mechanisms, such as rf losses locally enhanced by magnetic impurities, requires further investigation.

The poor rf performance and large density of pits observed in the large-grain cavity described in this study is fairly unusual. For example, we did an optical inspection of four large-grain single-cell cavities made from the same ingot material with RRR $\sim 500$ from Heraeus. These cavities were also etched by $\mathrm{BCP}$ and heat treated at $600^{\circ} \mathrm{C} / 10 \mathrm{~h}$ and had high $Q$ values up to quench fields of about $150 \mathrm{mT}$ [22]. The optical inspection did not reveal such a high density of etch pits as found in the large-grain cavity in this study. The resolution of the optical system used for the inspection is about $3 \mu \mathrm{m}$ at $56 \mathrm{~cm}$ observing distance. The cause for the high density of pits observed in the single-cell cavity used for this study is, at this point, not yet clear.

\section{CONCLUSION}

The analysis of the surface topography of samples cut from a large-grain single-cell SRF cavity characterized by anomalous nonlinear losses (a strong medium-field $Q$ slope) revealed a high density of etch pits. These were found to be distributed both along grain boundaries and within grains, the latter case possibly being related to the presence of lattice defects, such as dislocations, or impurities. Etch pits such as those observed in this study are a commonly recognized phenomenon with chemical etching of crystalline materials. The analysis of the local average misorientation angle distributions within grains showed a correlation between higher misorientation angles and higher density of etch pits. This suggests dislocations as nucleation sites for etch pits. A higher average density of etch pits was found to be correlated with higher rf losses. Because a larger aspect ratio of the pits would be required to explain the origin of the rf losses at low field than we observed, local magnetic field enhancement at the pits' sharp edges seems to be a secondary mechanism.

Further analysis of the samples by PCT done at Argonne National Laboratory seems to indicate that the origin of the anomalous rf losses is related to the presence of magnetic impurities in the oxide or at the metal/oxide interface at the pit locations. In conclusion, it would be worth exploring if the pits were somehow caused by ingot impurities or only by crystal lattice defects after deformation. If their origin is better understood, modifications to the process of metallurgy purification, mechanical deformation, or heat treatment might reduce the etch pit density in the final surface, and thus may improve the cavity rf performance.

\section{ACKNOWLEDGMENTS}

We thank G. Slack for the careful cavity dissection. We acknowledge Dr. M. Y. Zhu and the College of William \& Mary for their support on the SEM experiment. We appreciate Olga Trofimova for carefully counting the pit density. We also thank Dr. R. Cooks for his EBSD survey of all samples and Dr. A. Wu at JLab for the profilometry measurements. We would like to acknowledge Dr. C. Reece at JLab for supporting this activity. This manuscript has been authored by Jefferson Science Associates, LLC under U.S. DOE Contract No. DE-AC05-06OR23177. 
[1] L. Lilje, C. Antoine, C. Benvenuti, D. Bloess, J.-P. Charrier, E. Chiaveri, L. Ferreira, R. Losito, A. Matheisen, H. Preis, D. Proch, D. Reschke, H. Safa, P. Schmüser, D. Trines, B. Visentin, and H. Wenninger, Nucl. Instrum. Methods Phys. Res., Sect. A 516, 213 (2004).

[2] B. Visentin, in Proceedings of the 11th Workshop on RF Superconductivity, Travemünde, 2003, edited by D. Proch (DESY, Hamburg, Germany, 2004), TuO01; G. Ciovati, Physica C 441, 44 (2006).

[3] C. Benvenuti, S. Calatroni, I. E. Campisi, P. Darriulat, M. A. Peck, R. Russo, and A.-M. Valente, Physica C 316, 153 (1999).

[4] P. Kneisel, G. R. Myneni, G. Ciovati, J. Sekutowicz, and T. Carneiro, in Single Crystal-Large Grain Niobium Technology: Proceedings of the International Niobium Workshop, Araxá, Brasil, 2006, AIP Conf. Proc. No. 927, edited by G. R. Myneni, T. Carneiro, and A. Hutton (AIP, New York, 2007), p. 84.

[5] G. Ciovati, R. Flood, C. Grenoble, L. King, P. Kneisel, M. Morrone, and M. Snyder, Jefferson Lab Technical Note TN-05-59, 2005.

[6] A. Romanenko, Ph.D. thesis, Cornell University, 2009.

[7] V. Randle, Mater. Sci. Technol. 26, 253 (2010).

[8] D. Wolf, Mater. Sci. Eng. 107, 3 (1989).

[9] D. G. Brandon, Acta Metall. 14, 1479 (1966).

[10] $\mathrm{T}$ Furuhara and T. Maki, J. Mater. Sci. 40, 919 (2005).

[11] K Sangwal, Etching of Crystals. Theory, Experiment, and Application, Defects in Solids (Elsevier, New York, 1987), Vol. 15.

[12] B. C. Cai, A. Dasgupta, and Y. T. Chou, J. Less-Common Met. 90, 37 (1983).

[13] P. R. V. Evans, J. Less-Common Met. 6, 253 (1964).
[14] T. R. Bieler, N. T. Wright, F. Pourboghrat, C. Compton, K. T. Hartwig, D. Baars, A. Zamiri, S. Chandrasekaran, P. Darbandi, H. Jiang, E. Skoug, S. Balachandran, G. E. Ice, and W. Liu, Phys. Rev. ST Accel. Beams 13, 031002 (2010).

[15] L. N. Brewer, D. P. Field, and C. C. Merriman, in Electron Backscatter Diffraction in Materials Science, edited by A. J. Schwartz, M. Kumar, and D. P. Field (Springer, New York, 2009).

[16] A. Romanenko and H. Padamsee, Supercond. Sci. Technol. 23, 045008 (2010).

[17] G. Ciovati, G. Myneni, F. Stevie, P. Maheshwari, and D. Griffis, Phys. Rev. ST Accel. Beams 13, 022002 (2010).

[18] S. Berry, C. Antoine, and M. Desmons, in Proceedings of the 2004 European Particle Accelerator Conference, Lucerne, Switzerland, 2004, edited by J. Chrin, Ch. Petit-Jean-Genaz, J. Poole, C. Prior, and H.-A. Synal (PSI, Villigen, Switzerland, 2004), p. 1000.

[19] V. Shemelin and H. Padamsee, Tesla Technology Collaboration Report No. 2008-07.

[20] J. Knobloch, R. L. Geng, M. Liepe, and H. Padamsee, in Proceedings of the 9th Workshop on RF Superconductivity, Santa Fe, 1999, edited by F. Krawczyk (LANL, Los Alamos, NM, 2000), p. 77.

[21] T. Proslier, J. Norem, J. Elam, M. Pellin, J. Zasadzinski, G. Ciovati, and P. Kneisel, in Proceedings of the 14th International Conference on RF Superconductivity, Berlin, Germany, 2009, edited by M. Abo-Bakr, B. Kuske, A. Liebezeit, S. Voronenko, and V. Schaa (BESSY, Berlin, Germany, 2009), p. 137.

[22] P. Kneisel, in Proceedings of the 13th Workshop on RF Superconductivity, Beijing, China, 2007, edited by J. Hao, S. Huang, and K. Zhao (Peking University, Beijing, China, 2007), p. 728. 